\title{
Maîtrise d'écoulement diphasique dans les échangeurs à plaques
}

\author{
Mastering a two phase flow in plate exchangers
}

par A. Bailly et R. Jurkowski,

CIAT, Culoz

In order to obtain maximum performances in the exchanger, it must be ensured an uniform distribution of fluids between the diverse channels in the case of liquid-liquid flow (one phase flow) or of evaporation (two phase flow).

The distribution of a one phase flow is almost mastered but the two phase flow in a plate exchanger collector is setting some problems. It is needed to study flow rate in the inlet pipe and the mixing distribution in the collector.

Modelling with the CFD method is a promising way of research which may help to understand the phenomena inside the collector.

\section{I $\square$ INTRODUCTION}

Le marché des échangeurs présente un intérêt croissant pour les échangeurs à plaques brasées, qui concurrencent de plus en plus les échangeurs tubulaires pour des puissances toujours plus élevées.

Cependant leur potentiel de développement dans le domaine des machines frigorifiques se voit limité par quelques phénomènes, encore mal maîtrisés aujourd'hui, qui freinent leur expansion.

Ce type d'échangeur est composé de plaques superposées entre elles, qui après le brasage forment deux circuits composés chacun de plusieurs canaux parallèles et parcourus :

- d'un côté par un fluide à refroidir (écoulement monophasique),

- et de l'autre côté par un fluide à évaporer.

Ce fluide à évaporer, dit réfrigérant, rentre dans l'échangeur à partir d'un collecteur et se trouve en état diphasique, c'est-à-dire mélange de liquide et de vapeur.

Afin d'obtenir les performances maximales de l'échangeur, dans des conditions de fonctionnement données, nous savons qu'il est indispensable d'assurer une répartition uniforme des fluides entre les différents canaux.

Dans l'état actuel, la distribution d'un écoulement monophasique (caloporteur) reste pratiquement maîtrisée, par contre le phénomène de la distribution d'un écoulement diphasique à l'entrée de l'évaporateur à plaques pose quelques problèmes.

C'est ce soutirage successif du mélange diphasique dans le collecteur d'entrée, qui reste un des plus importants à maîtriser.
Donc, l'objectif de tous les travaux de recherche consiste à inventer et placer dans le collecteur d'entrée un dispositif de distribution permettant d'alimenter d'une manière homogène chaque canal de l'échangeur.

Afin de résoudre les problèmes évoqués ci-dessus, une triple approche semble être nécessaire : visualisation, simulations numériques de type CFD et expérimentation qui permet d'assurer la validation des résultats obtenus.

\section{DISTRIBUTION DIPHASIQUE}

Suite aux nombreux essais et calculs déjà effectués, nous savons que la bonne répartition du mélange diphasique dépend de plusieurs paramètres géométriques et de conditions optimales de fonctionnement :

Paramètres géométriques-exemples :

- la dureté de la plaque,

- le nombre de plaques,

- le diamètre et la rugosité du collecteur,

- la distance entre le détendeur et l'entrée du collecteur,

- le diamètre de tubulure,

- etc.

Conditions de fonctionnement - exemples :

- le type de réfrigérant,

- la pression de saturation,

- la vitesse et le titre massique à l'entrée,

- la vitesse massique à la sortie des canaux,

- etc.

Le schéma simplifié d'écoulement se trouve sur la figure 1.

Donc, globalement nous pouvons dire que tous ces para- 
mètres ont une influence sur le régime d'écoulement diphasique à l'intérieur du collecteur qui résulte de la présence simultanée de deux conditions contradictoires :

1- diminution de la vitesse homogène d'écoulement diphasique à l'entrée pour améliorer la répartition de débit entre les différents canaux par analogie avec l'écoulement monophasique [1].

2- augmentation de la vitesse homogène d'écoulement pour éviter la séparation de phases.

Notons que pour un titre massique à l'entrée de l'ordre de $20 \%$, le taux de vide peut dépasser facilement $90 \%$, si nous appliquons la corrélation de Premoli avec un coefficient de glissement de phases égal à 1 .

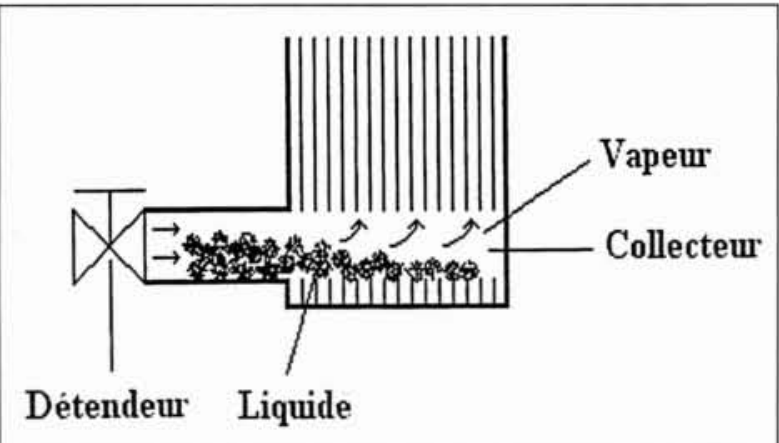

1. Schéma d'écoulement - répartition simplifiée du liquide et de la vapeur.

\subsection{Approche de visualisation}

Si nous analysons le régime d'écoulement diphasique à partir des cartes d'écoulement existantes [2], [3], [4], (établies pour les régimes stabilisés), nous pouvons constater que dans nos cas, à l'entrée nous avons bien un régime d'écoulement de type stratifié ou stratifié ondulé.

Remarquons cependant que tous les tests de visualisation réalisés jusqu'à maintenant n'ont pas pu démontrer clairement l'état diphasique en question puisque nous sommes loin d'un régime d'écoulement stabilisé.

Effectivement, l'influence d'un effet de jet, créé par la présence approchée d'un détendeur thermostatique et l'évaporation en flash sont des éléments fortement perturbateurs.

Néanmoins, nous avons pu observer (essais avec une tubulure en verre) davantage de liquide présent dans la partie basse de la tubulure et un écoulement de type dispersé (brouillard) dans la partie supérieure.

L'utilisation d'une caméra rapide et l'éclairage spécifique s'avèrent indispensables dans ce type d'approche.

Remarquons de plus que toutes les tentatives de visualisation ont pu être effectuées uniquement sur la tubulure d'entrée ; or les phénomènes déterminants se déroulent surtout à l'intérieur du collecteur.

\subsection{Approche CFD}

Avec cet outil performant, nous pouvons analyser les phénomènes de distribution qui se déroulent non seulement dans les collecteurs mais également au sein des plaques.

A l'intérieur du collecteur, les deux phases (liquide et vapeur ) n'ont guère d'autres possibilités que d'alimenter les canaux existants.

Cependant, nous pouvons imaginer deux scénarios :

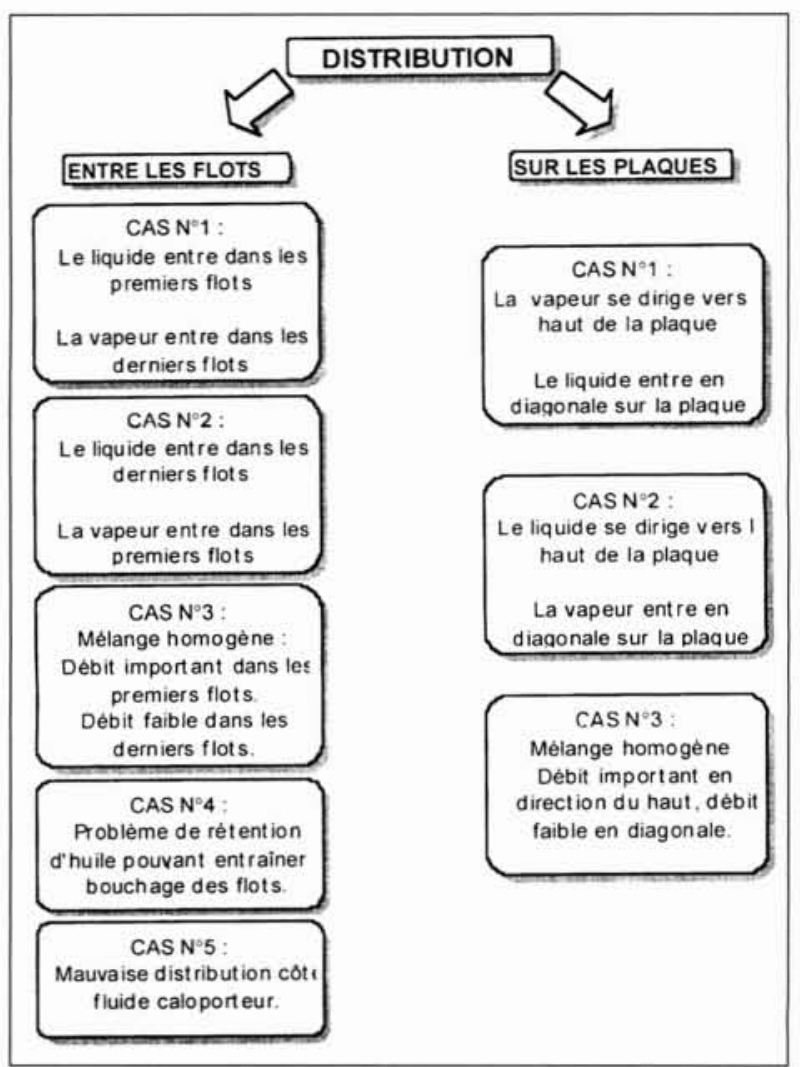

2. Différents cas de distribution entre les flots et sur les plaques.

1. Le liquide entre par soutirage dans la première partie des canaux et la vapeur dans les canaux suivants (voir fig. 2 cas 1),

2. C'est la vapeur qui rentre dans les premiers canaux et le liquide par la force d'inertie remplit les derniers canaux (voir fig. 2 cas 2).

Remarquons qu’en réalité, la vérité peut être plus complexe et que nous pourrions avoir les deux phénomènes simultanément sans pour autant obtenir une répartition uniforme de la vapeur et du liquide.

De plus comme nous avons précisé ci-dessus, le problème de répartition de mélange diphasique entre les canaux peut être accompagné d'un problème de distribution de ce mélange au sein de la plaque même (fig. 3).

Cette approche peut effectivement apporter un gain considérable au niveau du temps et des moyens mis en jeux, si nous arrivons à modéliser réellement l'écoulement diphasique en tant que tel :

- présence simultanée des gouttelettes et du liquide,

- accumulation du liquide,

- coalescence et dispersion des gouttelettes,

- déposition des gouttelettes à la paroi ou à l'interface liquide-vapeur,

- arrachement des gouttelettes de la paroi ou d'un film liquide,

- rebondissement des gouttelettes de la paroi ou entre elles.

Autrement dit les phénomènes de transfert et d'échange de masse entre les différents régimes d'écoulement (présents simultanément) doivent être pris en compte. 


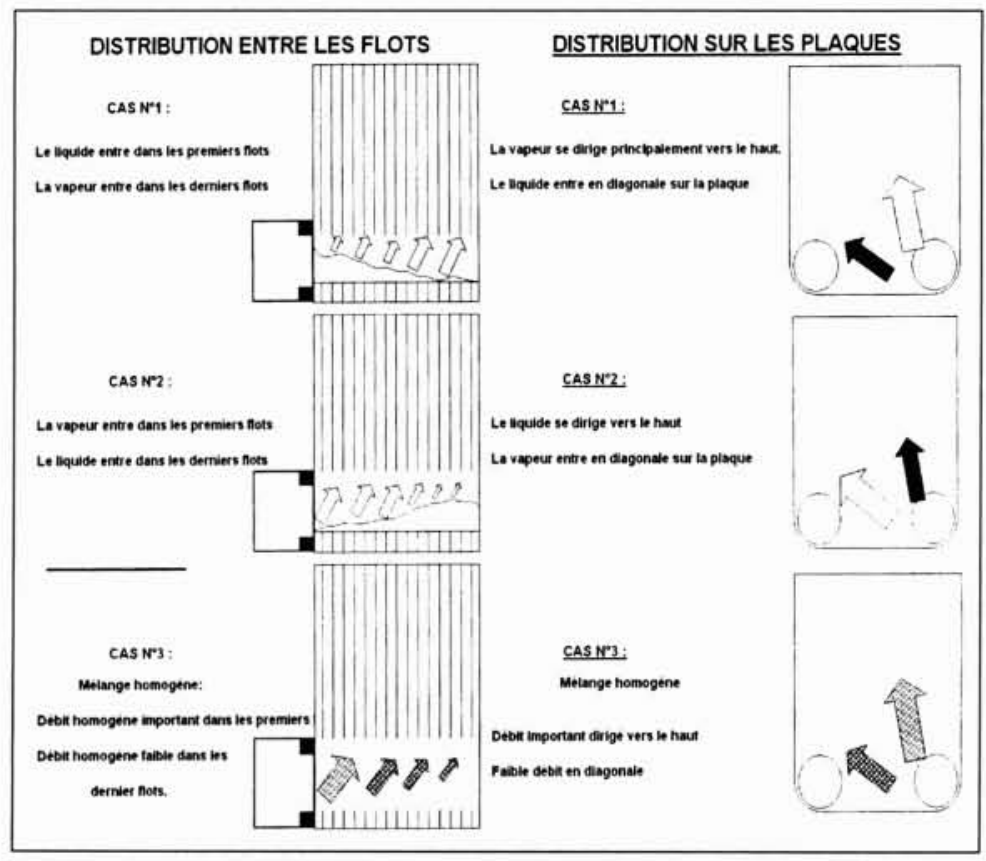

3. Illustration des différents cas de distribution entre les flots et sur les plaques.

\section{ENJEUX}

Actuellement, suite à l'expérience acquise dans ce domaine, nous pouvons constater que la distribution d'écoulement diphasique reste satisfaisante jusqu'à 25 canaux environ (ce qui correspond à 50 plaques), sans aucun élément additionnel permettant l'amélioration de la distribution (fig. 4).

Au-delà de ce nombre, c'est-à-dire si l'on augmente le nombre de plaques, nous assistons à une dégradation des performances de l'échangeur qui peut s'expliquer par le problème de mauvaise distribution provoqué entre autres par le phénomène de séparation des phases.

Alors il est absolument nécessaire de maîtriser la répartition uniforme d'écoulement diphasique, si nous voulons maximiser les performances de l'échangeur.

Plusieurs thèses [5],[6],[7],[8] et publications scientifiques [9] à [12] ont déjà essayé aborder ce sujet sur les plans expérimental et analytique. Il existe également quelques programmes numériques (Trio, Astrid, CFX, Fluidyn et autres) qui tentent de modéliser les écoulements diphasiques tridimensionnels .

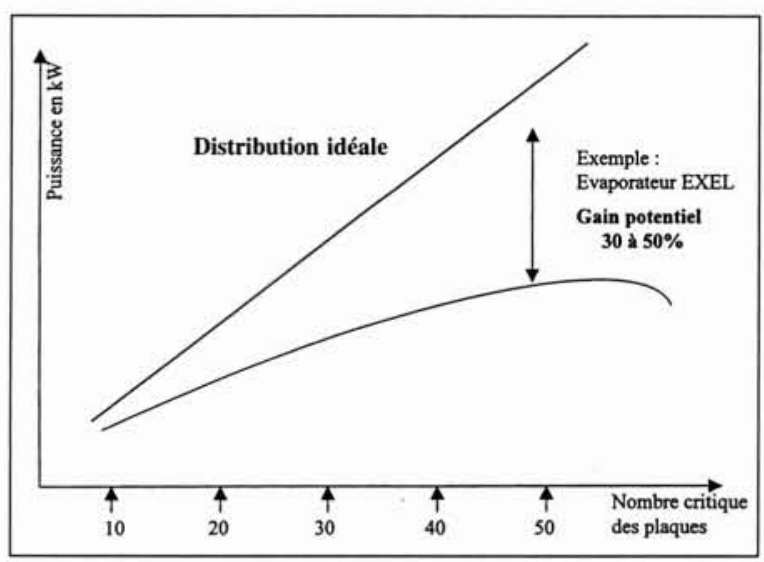

4. Évolution des performances d'un évaporateur à plaques brasées en fonction du nombre de canaux.
Mais il ne faut pas oublier que dans une machine frigorifique il existe également une circulation d'huile, plus ou moins miscible, nécessaire à la lubrification du compresseur.

Dans le cas de mauvaises conditions de fonctionnement, l'huile peut provoquer le bouchage des canaux par le phénomène de rétention.

C'est pratiquement une troisième phase, partiellement miscible avec le réfrigérant, qu'il faudrait prendre en compte afin de cerner le problème de la distribution dans sa globalité.

\section{IV $\square$ CONCLUSIONS}

Aujourd'hui l'apport de la démarche scientifique fait appel aux deux approches, modélisation et expérimentation, qui s'enrichissent mutuellement.

L'analyse non destructive par la simulation numérique reste une voie d'investigation possible. La mise au point de modèles, validés expérimentalement, devrait permettre la localisation des zones occupées préférentiellement par le gaz ou par le liquide et par conséquent, autoriser la prédiction d'évolution de taux de vide à l'intérieur du collecteur de l'évaporateur.

C'est une condition sine qua non pour un chercheur de développer les dispositifs permettant la maîtrise de phénomènes de distribution diphasique et pour un industriel d'accepter cette méthode comme une approche d'investigation fiable.

Le cas que nous venons de présenter est particulièrement difficile à traiter puisque les limites d'approche expérimentale sont bien évidentes (impossibilité de pénétrer à l'intérieur de l'échangeur) et nous savons tous que l'approche CFD reste encore très difficile

Mais, malgré tout, compte tenu du potentiel d'amélioration, nous restons dans l'attente, puisque le sujet mérite un investissement de la part des spécialistes de CFD.

\section{Références}

[1] B. ThoNON, Étude et optimisation de la distribution du fluide dans un échangeur de chaleur à plaques, Thèse Université de Nancy 1991.

[2] Ch. Marvillet ,B.Thonon, Problèmes de Distributions dans les échangeur, RPF 1996.

[3] J. M. Delhaye, Écoulements diphasiques, ENSHMG de Grenoble, 1995.

[4] HTFS Handbook vol 3 TM2.

[5] J. GRADECK, Structure de l'écoulement diphasique gaz-liquide dans les échangeurs à plaques corruguées, Thèse Université Nancy I, 1996.

[6] M. IDRISs, Étude de la distribution d'un écoulement diphasique entre deux passes d'un évaporateur tubulaire, Thèse INPL ,Grenoble.

[7] L. F. Mendes dE Moura, Étude de la redistribution d'un écoulement diphasique entre deux passes d'un échangeur de chaleur. Thèse INPG, Grenoble 1989.

[8] M. SouHaR, Étude de frottement pariétale dans les écoulements diphasiques en conduite verticale ...,Thèse INPL Nancy, 1979.

[9] J. M. DelhaYE, Écoulements polyphasiques et transferts, École d'été GUT, Pont à Mousson 1990.

[10] BANKHOFF et LEE, Survey of two phases flows, 1983.

[11] Haestronni, Multiphase flows, Hemisphere Publ.Corp ,1989.

[12] Bergles, Delhaye and al., Two phase flow and heat transfer in the power and process industries, Hemisphere Publ. Corp., 1981. 